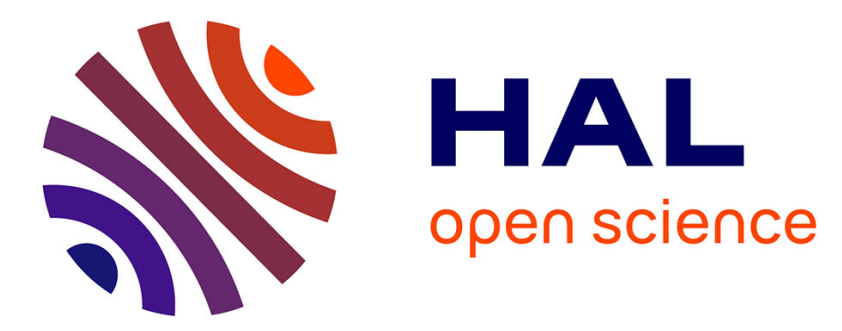

\title{
Estimation de la largeur de bande des formants à partir de la distribution cumulée du niveau sonore dans le spectre

\author{
R. Ruiz, C. Legros
}

\section{- To cite this version:}

R. Ruiz, C. Legros. Estimation de la largeur de bande des formants à partir de la distribution cumulée du niveau sonore dans le spectre. Journal de Physique IV Proceedings, 1994, 04 (C5), pp.C5-461-C5466. 10.1051/jp4:1994596 . jpa-00253091

\section{HAL Id: jpa-00253091 https://hal.science/jpa-00253091}

Submitted on 1 Jan 1994

HAL is a multi-disciplinary open access archive for the deposit and dissemination of scientific research documents, whether they are published or not. The documents may come from teaching and research institutions in France or abroad, or from public or private research centers.
L'archive ouverte pluridisciplinaire HAL, est destinée au dépôt et à la diffusion de documents scientifiques de niveau recherche, publiés ou non, émanant des établissements d'enseignement et de recherche français ou étrangers, des laboratoires publics ou privés. 


\title{
Estimation de la largeur de bande des formants à partir de la distribution cumulée du niveau sonore dans le spectre
}

\author{
R. RUIZ et C. LEGROS \\ Laboratoire d'Acoustique, Université Toulouse-Le Mirail, 5 allées Antonio Machado, 31058 Toulouse \\ cedex, France
}

\begin{abstract}
The measurement of a bandwith on a spectral envelope can be deduced from the Cumulative Spectrum Probability Diagram (CSPD) of sound level. First, it is necessary that the spectrum be sampled in frequency and the sound level scale be divided in equal amplitude classes. Then, it can be demonstrated that a simple relation between bandwith and statistical index of the Cumulative Spectrum Probability Diagram exists.This property is verified thanks to a known mathematically frequency function (i.e an envelope spectrum). Bandwiths directly obtained from the function are compared to estimated ones issued from the CSPD. This method can be applied to the estimation of the formant bandwiths on the envelope spectrum of a vowel signal.
\end{abstract}

Résumé : L'estimation d'une largeur de bande sur l'enveloppe d'un spectre peut être déduite du diagramme représentant la distribution cumulée du niveau sonore. Il existe alors une relation simple entre largeur de bande et indice statistique du diagramme. L'objet de cette communication est d'établir la relation entre les deux grandeurs puis d'en proposer une vérification expérimentale. Nous envisagerons enfin son application à la mesure de la largeur de bande des formants d'une voyelle.

\section{1 - Le Diagramme de Probabilité Spectrale Cumulée du niveau sonore.}

Considérons l'enveloppe d'un spectre comportant $N$ fréquences discrètes entre $f_{\min }$ et $f_{\max }(\mathrm{Hz})$, l'histogramme du niveau sonore peut être défini comme suit :

$$
\mathrm{P}\left(\mathrm{L}_{\mathrm{i}}\right)=\left(\frac{\mathrm{N}_{\mathrm{i}}}{\mathrm{N}}\right)
$$

où $\mathrm{N}_{\mathrm{i}}$ désigne le nombre de fréquences discrètes (ou raies) pour lesquelles le niveau sonore appartient à la classe de niveau de valeur centrale $\mathrm{L}_{\mathrm{i}}$.

$\mathrm{P}\left(\mathrm{L}_{\mathbf{i}}\right)$ désigne donc la proportion de fréquences discrètes comprises dans la classe de niveau $\mathrm{L}_{\mathbf{i}}$ et de largeur de classe $\Delta \mathrm{L}$.

La probabilité cumulée, notée $\mathrm{I}_{\mathbf{i}}$, correspondant au niveau $\mathrm{L}_{\mathbf{i}}$ est définie par la relation suivante :

$$
\mathrm{I}_{\mathrm{i}}=1-\sum_{j=1}^{i} P\left(L_{j}\right)
$$


Elle exprime le cumul des proportions des classes dont le niveau est inférieur ou égal à $L_{\mathrm{i}}$.

Le diagramme représentant $I_{i}$ en fonction de $L_{i}$ est appelé Diagramme de Probabilité Spectrale Cumulée du niveau sonore (DPSC), et $\mathrm{I}_{i}$ est nommé indice statistique.

La figure 1 est un exemple de calcul du diagramme. On note que le DPSC résulte du lissage de l'histogramme cumulé par une régression polynomiale.

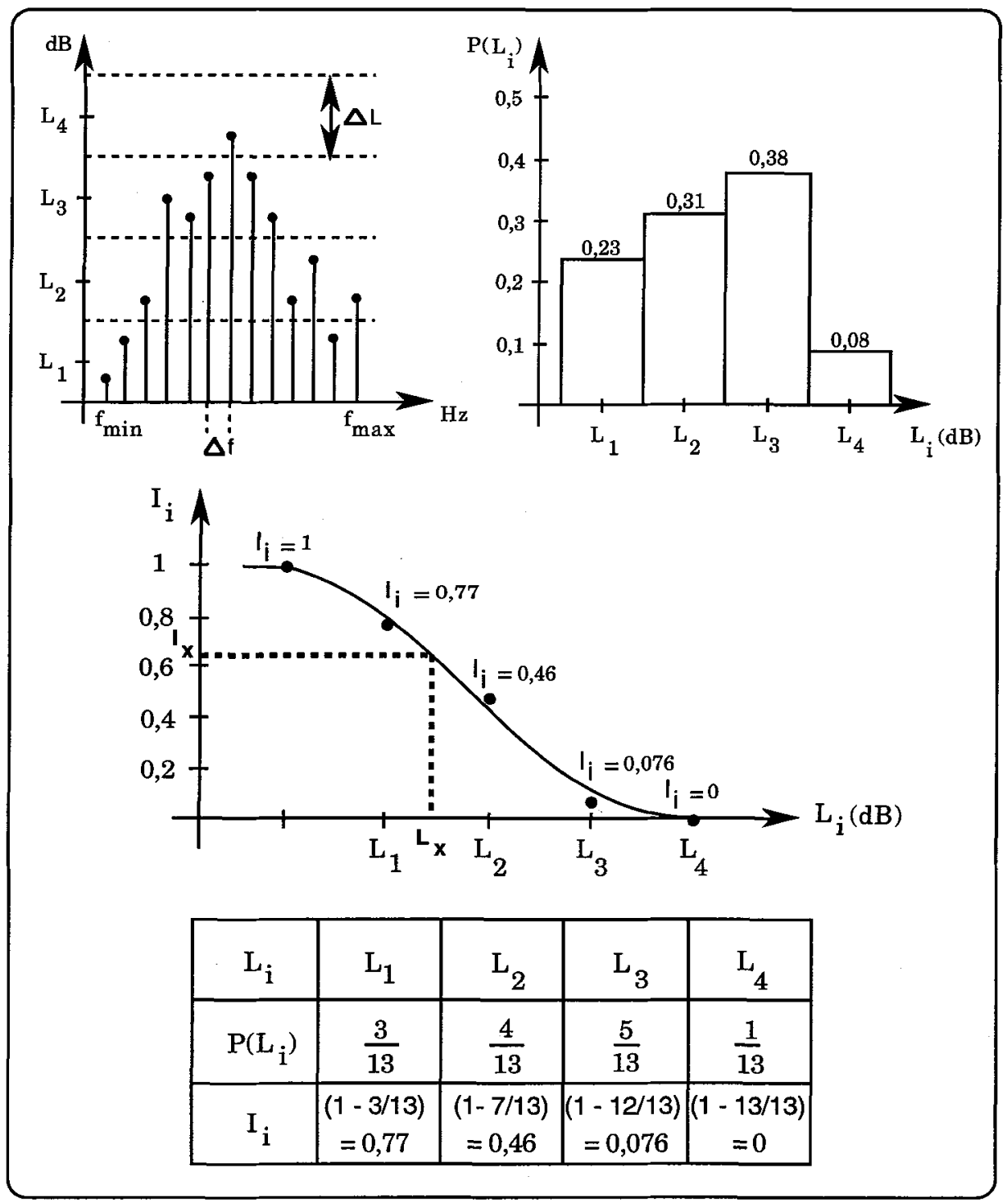

Figure 1: exemple de calcul du Diagramme de Probabilité Spectrale Cumulée du niveau sonore à partir de l'enveloppe d'un spectre échantillonnée en fréquence (13 raies). 


\section{2 - Estimation des largeurs de bande.}

Le Diagramme de Probabilité Spectrale Cumulée permet d'estimer les largeurs de bande. En effet (figure 1), $\mathrm{L}_{\mathrm{X}}$ est le niveau dépassé pour $\left(100 . \mathrm{I}_{\mathrm{X}}\right) \%$ du domaine de fréquence $\left(\mathrm{f}_{\max }-\mathrm{f}_{\min }\right)(\mathrm{Hz})$. Cette propriété résulte de la définition même de la probabilité cumulée.

Ainsi, on note dans l'exemple ci-dessus que le niveau $L_{2}$ est dépassé pour $46 \%$ de $\left(f_{\max }-f_{\min }\right)$ $(\mathrm{Hz})$. Le niveau $\mathrm{L}_{4}$ n'est jamais dépassé $(\mathrm{I}=0)$ et les classes de niveau inférieures à $\mathrm{L}_{1}$ sont toujours dépassées $(I=1)$.

Ces considérations peuvent être traduites en terme de largeur de bande. Le niveau $\mathrm{L}_{3}$ est dépassé pour $\left[0.46\left(f_{\max }-f_{\min }\right)\right]$. Autrement dit, la largeur de bande à $-\left(L_{4}-L_{2}\right)$ (dB) vaut $\left[0.46\left(f_{\max }-f_{\min }\right)\right](H z)$.

Plus généralement, la largeur de bande $\mathrm{B}(\mathrm{en} \mathrm{Hz}) \mathrm{à}-\left(\mathrm{L}_{\max }-\mathrm{L}_{\mathrm{X}}\right)(\mathrm{dB})$ s'écrit:

$$
B=I_{X}\left(f_{\max }-f_{\min }\right)
$$

où $\mathrm{L}_{\max }$ est la valeur centrale de la classe du niveau maximal atteint dans le domaine de fréquence étudié, soit de $f_{\min } \grave{a} f_{\max }$.

Pour $\mathrm{I}_{\mathrm{X}}=0$, c'est-à-dire pour $\mathrm{L}_{\mathrm{X}}=\mathrm{L}_{\max }$, on a $\mathrm{B}=0 \mathrm{~Hz}$, et pour $\mathrm{I}_{\mathrm{X}}=1$, c'est-à-dire pour , $\mathrm{L}_{\mathrm{X}} \leq\left(\mathrm{L}_{\min }-\frac{\Delta \mathrm{L}}{2}\right)$, on a $\mathrm{B}=\left(\mathrm{f}_{\max }-\mathrm{f}_{\min }\right)$.

Enfin, pour $\mathrm{L}_{\mathrm{X}}$ tel que $\left(\mathrm{L}_{\max }-\mathrm{L}_{\mathrm{X}}\right)=3 \mathrm{~dB}, \mathrm{~B}$ désigne la largeur de bande classique à $-3 \mathrm{~dB}$.

\section{Vérification expérimentale.}

Afin de vérifier la relation (3), nous avons simulé l'enveloppe d'un spectre en forme de parabole. Ceci dans le but d'appliquer la méthode à la mesure des largeurs de bande des formants. Son équation est de la forme $L(f)=a f^{2}+b f+c$, où $L$ et $f$ désignent respectivement le niveau et la fréquence et $a, b, c$ sont des constantes.

La largeur de bande à $-\left(\mathrm{L}_{\max }-\mathrm{L}_{\mathbf{X}}\right) \mathrm{dB}$ s'écrit alors (en $\left.\mathrm{Hz}\right)$ :

$$
B=\left|\frac{\sqrt{b^{2}-4 a\left(c-L_{x}\right)}}{a}\right|
$$

La comparaison entre la vraie valeur de B donnée par la relation (4), et son estimation par la relation (3) est obtenue en calculant la valeur absolue de la différence de ces deux valeurs.

Il est bien sûr nécessaire de procéder au tracé du DPSC en ayant au préalable échantillonné en fréquence l'enveloppe du spectre $\mathrm{L}(\mathrm{f})$ (résolution $\Delta \mathrm{f}$ ).

On obtient les courbes de la figure 2. Elles représentent l'erreur d'estimation des largeurs de bande en fonction de la différence $\left(L_{\max }-L_{X}\right)$ en dB. Ces courbes ont été établies pour une largeur de classe de niveau $\Delta \mathrm{L}=1 \mathrm{~dB}$ et une résolution fréquentielle $\Delta \mathrm{f}$ de $10 \mathrm{~Hz}, 1 \mathrm{~Hz}$ et $0,1 \mathrm{~Hz}$.

On note que l'erreur commise est d'autant plus petite que $\Delta \mathrm{f}$ et $\Delta \mathrm{L}$ sont conjointement moins élevés. Dans ces conditions, elle peut devenir faible (inférieure à $1 \mathrm{~Hz}$ ). 


\section{Erreur $(\mathrm{Hz})$}

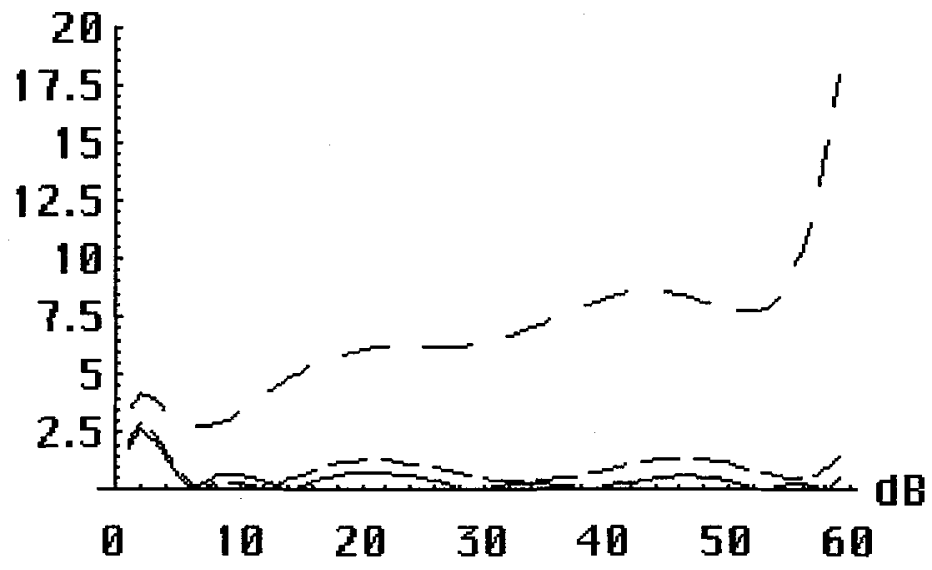

Figure 2: Exemple d'erreur d'estimation (en $\mathrm{Hz}$ ) des largeurs de bande par la méthode du DPSC avec une largeur de classe de niveau: $\Delta \mathrm{L}=1 \mathrm{~dB}$ et une résolution fréquentielle:

$$
\begin{aligned}
&--1: \Delta \mathrm{f}=10 \mathrm{~Hz} \\
&--1 \Delta \mathrm{f}=1 \mathrm{~Hz} \\
&: \Delta \mathrm{f}=0.1 \mathrm{~Hz}
\end{aligned}
$$

\section{4-Application au cas du signal de voyelle.}

Cette méthode d'estimation des largeurs de bande s'applique à l'enveloppe du spectre échantillonnée en fréquence. Elle est donc adaptée à la reconstruction spectrale à partir des coefficients de prédiction linéaire. Ceux-ci sont ordonnés dans le temps puis on leur applique une Transformée de Fourier Rapide (T.F.R). L'enveloppe est donc définie par l'amplitude des raies issues de la T.F.R [1]. C'est à partir de cet ensemble fini de raies qu'est effectué le calcul et le tracé du DPSC [2].

Sans précaution particulière, une largeur de bande déduite du diagramme peut ne pas correspondre à celle d'un formant, mais à la somme des largeurs de bande des résonances du domaine de fréquence considéré. Avant de calculer le DPSC, il est donc nécessaire de limiter le domaine à l'intervalle de fréquence autour de la résonance étudiée. On en déduira alors toutes les largeurs de bande du formant considéré.

Enfin, sous réserve que la résolution fréquentielle associée à l'échantillonnage de l'enveloppe spectrale, et la largeur des classes de niveau soient suffisamment faibles, les erreurs d'estimation seront du même ordre de grandeur que celles indiquées par la simulation mathématique précédente.

\section{5 - Conclusion.}

La méthode proposée présente l'avantage d'être adaptée aux techniques de modélisation AutoRégressive du signal, mais elle est en contrepartie lourde à mettre en oeuvre: calcul de l'histogramme cumulé puis régression polynomiale avec des conditions strictes de résolution fréquentielle et de largeur de classe d'amplitude. Elle confère cependant à l'estimation de la distribution cumulée du niveau dans le spectre autant d'informations que l'enveloppe spectrale dont elle est issue.

\section{Références.}

[1] MARKEL J.D. and GRAY A.H. Jr., Linear prediction of speech, (Springer-Verlag, 1982).

[2] RUIZ R., Contribution à l'étude de l'influence d'une charge de travail sur les caractéristiques acoustiques de la voix, Thèse de doctorat de l'Université Toulouse 3, spécialité acoustique, 29 Juin 1991. 